\title{
Phenotyping-based treatment improves obstructive sleep apnea symptoms and severity: a pilot study
}

\author{
Ludovico Messineo $^{1} \cdot$ Roberto Magri $^{1} \cdot$ Luciano Corda $^{1} \cdot$ Laura Pini $^{1} \cdot$ \\ Luigi Taranto-Montemurro $^{2}$. Claudio Tantucci ${ }^{1}$
}

Received: 4 October 2016 /Revised: 29 November 2016 / Accepted: 16 February 2017 / Published online: 25 February 2017

(C) Springer-Verlag Berlin Heidelberg 2017

\begin{abstract}
Background Obstructive sleep apnea is a common disorder characterized by multiple pathogenetic roots. Continuous positive airway pressure (CPAP) is almost always prescribed as the first-line treatment to all patients regardless of the heterogeneous pathophysiology, because it mechanically splints the airways open and reduces the collapsibility of the upper airway. Despite its high efficacy, CPAP is burdened by poor adherence and compliance rates. In this pilot study, we treated OSA patients with composite approaches different than CPAP, tailoring the therapeutic choice on OSA phenotypic traits.

Methods We used the CPAP dial down technique to assess phenotypic traits in eight OSA patients with $\mathrm{BMI}<35$. According to these traits, patients received personalized therapies for 2-week period, after which we ran a second polygraphy to compare apnea-hypopnea index (AHI) before and after therapy.

Results Two weeks of combined behavioral and pharmacological therapy induced a significant reduction in mean AHI, which dropped from $26 \pm 15$ at baseline to $9 \pm 7$ post-treatment $(p=0.01)$. Furthermore, there was a significant reduction in mean ODI $(p=0.03)$ and subjective sleepiness $(p=0.01)$ documented by Epworth Sleepiness Scale (ESS) from baseline to post-treatment recordings.
\end{abstract}

Ludovico Messineo

ludovico.messineo@yahoo.it

1 Respiratory Medicine and Sleep Laboratory, Department of Experimental and Clinical Sciences, University of Brescia and Spedali Civili, Piazzale Spedali Civili 1, 25000 Brescia, Italy

2 Division of Sleep and Circadian Disorders, Departments of Medicine and Neurology, Brigham \& Women's Hospital \& Harvard Medical School, Boston, MA, USA
Conclusions Treating OSA patients with a personalized combination of pharmacological and behavioral therapies according to phenotypic traits leads to a significant improvement in AHI, ODI, and subjective sleepiness.

Keywords OSA phenotyping · OSA pharmacological therapy $\cdot$ OSA behavioral therapy $\cdot$ Alternative treatment for sleep apnea

\section{Introduction}

Obstructive apnea syndrome (OSA) is a pandemic disorder with a prevalence of $22 \%$ in men and $17 \%$ in women [1], and continuous positive airway pressure (CPAP) is the standard way to treat this disease. However, OSA pathogenesis is complex and heterogeneous and, recently, at least four pathogenetic traits were demonstrated to be involved in determining OSA [2]: (1) high upper airway (UA) collapsibility, (2) low upper airway gain (UAG), (3) low arousal threshold (AT), and (4) high instability of breathing control (Loop Gain). The interaction among these traits in provoking OSA defines the individual OSA phenotypes [2].

The UA collapsibility is believed as doing the lion's share in determining OSA. It is due to an altered congenital or acquired anatomy which results in an increased collapsibility of the UA that can be measured as the critical closing pressure of the UA $\left(P_{\mathrm{CRIT}}\right)$. Despite obesity is often linked to an unfavorable anatomy [3, 4], about $30 \%$ of OSA patients are not obese [5] and it has been demonstrated that a high $P_{\text {CRIT }}$ justifies only $30 \%$ of OSA severity $[6,7]$. The UAG is the neuromuscular response of the UA dilator muscles to avoid pharyngeal collapse [8], and it depends on the integrity of the UA muscles and on their capability to be triggered by stimuli like increase in $\mathrm{PaCO}_{2}$ and negative pharyngeal pressure while the 
subject is asleep [9]. Therefore, the longer the time for recruiting UA muscles by these stimuli, the greater will be their activation. In patients with a low arousal threshold, the premature occurrence of an arousal from sleep can limit the neuromuscular compensation of UA muscles, leading to the development of apneas and hypopneas [10]. Lastly, loop gain (LG) is the expression of respiratory center activity, and it is measured as the response to a ventilatory disturbance [11]: the higher the response, the higher the system instability and the likelihood of OSA [12].

CPAP successfully treats OSA by mechanical UA opening regardless of patient's phenotype. Despite being highly effective in treating OSA, CPAP has poor adherence [13] and compliance rates [14].

Thus, OSA differentiation based on predominant pathogenic traits has been recently proposed in order to define therapeutic targets [15], but, to our knowledge, no study about multiple treatments has been performed to validate this approach.

So, we performed a pilot study to assess the treatment response of OSA patients to a composite therapeutic approach different than CPAP, driven by the subject's phenotype.

\section{Methods}

\section{Subjects and techniques}

This investigation was performed at the Respiratory Medicine Unit of Spedali Civili Hospital, Brescia, Italy. Eight subjects diagnosed with OSA of any severity (AHI $>5$ events/h) were recruited from August 2015 to March 2016 in our sleep Laboratory. Diagnosis was made with an all-night cardiorespiratory polygraphy (Embletta, Embla, Broomfield, Colorado, USA) according to AASM criteria [16], with at least $4 \mathrm{~h}$ of sleep and $>50 \%$ of events obstructive. Exclusion criteria were BMI $\geq 35 \mathrm{~kg} / \mathrm{m}^{2}$, acute o chronic usage of antidepressants, hypnotics or sedatives, neuromuscular diseases, dysthyroidisms, sleep disorders other than OSA, and uncompensated diabetes. Patients with moderate-to-severe obesity were excluded in order to reduce the impact of high collapsibility on the severity of OSA.

For all patients, the following data were recorded: age, sex, BMI, neck and waist circumference, Epworth sleepiness scale (ESS), apnea-hypopnea index (AHI), oxygen desaturation index (ODI), average and minimum oxyhemoglobin saturation, average oxyhemoglobin desaturation, and sleep time percentage under $90 \%$ of oxyhemoglobin saturation (CT90). Subsequently, all patients underwent a complete polysomnography in a nighthospital regimen for detecting the phenotype within 1 week from the first test, with recordings of electroencephalography (EEG) with International 10-20 system, electrooculography (EOG), chin and leg electromyography (EMG), airflow through a pneumotachograph (Hans-Rudolph, Kansas City, Missouri,
USA) and a pressure transducer (Validyne, Northridge, California, USA), thoracic and abdominal bands, and oxyhemoglobin saturation. Patients breathed through a nasal mask, connected to the pneumotachograph and to a CPAP, delivering pressure from 0 to $30 \mathrm{cmH}_{2} \mathrm{O}$. Subjects were asked to sleep in supine position. Once the patient was asleep, CPAP was set with the pressure capable of eliminating all obstructive events, inspiratory flow limitation and snoring. Then, following Wellman and coworkers' simplified method of phenotyping [17], all nightlong CPAP maneuvers were performed, in order to evaluate the patient's response to these ventilatory disturbances and, consequently, to determine $\mathrm{UAG}, \mathrm{AT}, \mathrm{LG}$, and $P_{\mathrm{CRIT}}$ for settling patients' phenotype.

Based on the computed variables, a phenotype graph was drawn for each patient of the study following the phenotyping night. After the analysis, each patient was referred again to our laboratory to start a personalized therapy for 2 weeks, according to his previously determined defective pathogenetic traits. Two drugs and two behavioral approaches were proposed, alone or in association: trazodone $75 \mathrm{mg}$ once daily was administered $30^{\prime}$ before sleep to those with low AT (AT $<10 \mathrm{~L} / \mathrm{min}$ ); acetazolamide $250 \mathrm{mg}$ twice daily was administered to those with high steady state $\mathrm{LG}(\mathrm{LG}>2)$; compression stockings were suggested to those with high collapsibility $\left(V_{0, \text { PASSIVE }}<1 \mathrm{~L} / \mathrm{min}\right)$, adding a hypocaloric diet (1200 kcal divided as 55\% complex carbohydrates, $20 \%$ protein, $25 \%$ fat of which $<7 \%$ saturated fat) when collapsibility was particularly low $\left(V_{0, \text { PASSIVE }} \leq 0.25 \mathrm{~L} / \mathrm{min}\right)$.

Finally, on the last day of the tailored therapy, any changes in AHI, ODI, mean and minimum oxyhemoglobin saturation, average oxyhemoglobin desaturation, and CT90 were investigated in all patients with another all-night cardiorespiratory polygraphy (Embletta, Embla, Broomfield, Colorado, USA). ESS after therapy was also documented.

In summary, the study design was divided in four steps: a basal home polygraphy, a phenotyping polysomnography, a medical examination which included the choice of an individualized therapy for each patient, and a home polygraphy after 2 weeks of such therapy.

This study was approved by local Ethic Committee. Informed written consent was obtained from all subjects.

\section{Data analysis}

Signals were sampled at $125 \mathrm{~Hz}$, recorded and analyzed with ProFusion 4 software (Compumedics Limited, Victoria, Australia). Overnight phenotyping polysomnography analyses were made using standard scoring criteria for sleep stages and arousals [16]. Only data from non-rapid eye movements (NREM) sleep were taken into account in the analysis: REM sleep periods and wake after sleep onset (WASO) periods were excluded from the analysis. 
Eupnoic ventilation $\left(V_{E}\right)$ was calculated as the average value after 1 min of stable breathing on therapeutic CPAP. UA collapsibility was estimated by the level of ventilation at $0 \mathrm{cmH}_{2} \mathrm{O}$ of CPAP when UA muscles are not activated. It was calculated as the mean value of ventilation after having dropped down CPAP from therapeutic level to $0 \mathrm{cmH}_{2} \mathrm{O}$ for four to five breaths ( $\left.V_{0, \text { PASSIVE }}\right)$, before returning at optimum CPAP. Then, the CPAP level which leads to an EEG arousal was calculated by lowering pressure by $1 \mathrm{cmH}_{2} \mathrm{O}$ every minute until an arousal occurred. Average ventilation of five flowlimited breaths before the arousal corresponds to the maximum reduction of ventilation tolerated by the asleep subject ( $V_{\text {AROUSAL }}$ ). If in the following minute, another arousal did not occur, CPAP was dropped again to $0 \mathrm{cmH}_{2} \mathrm{O}$ to calculate the ventilation at $0 \mathrm{cmH}_{2} \mathrm{O}$ of CPAP when UA muscles were maximally active $\left(V_{0, \mathrm{ACTIVE}}\right)$. Otherwise, from $V_{\mathrm{AROUSAL}}$, CPAP could be brought to optimum level (Dial Up), in order to find $\mathrm{LG}$, calculated as the ratio between the average
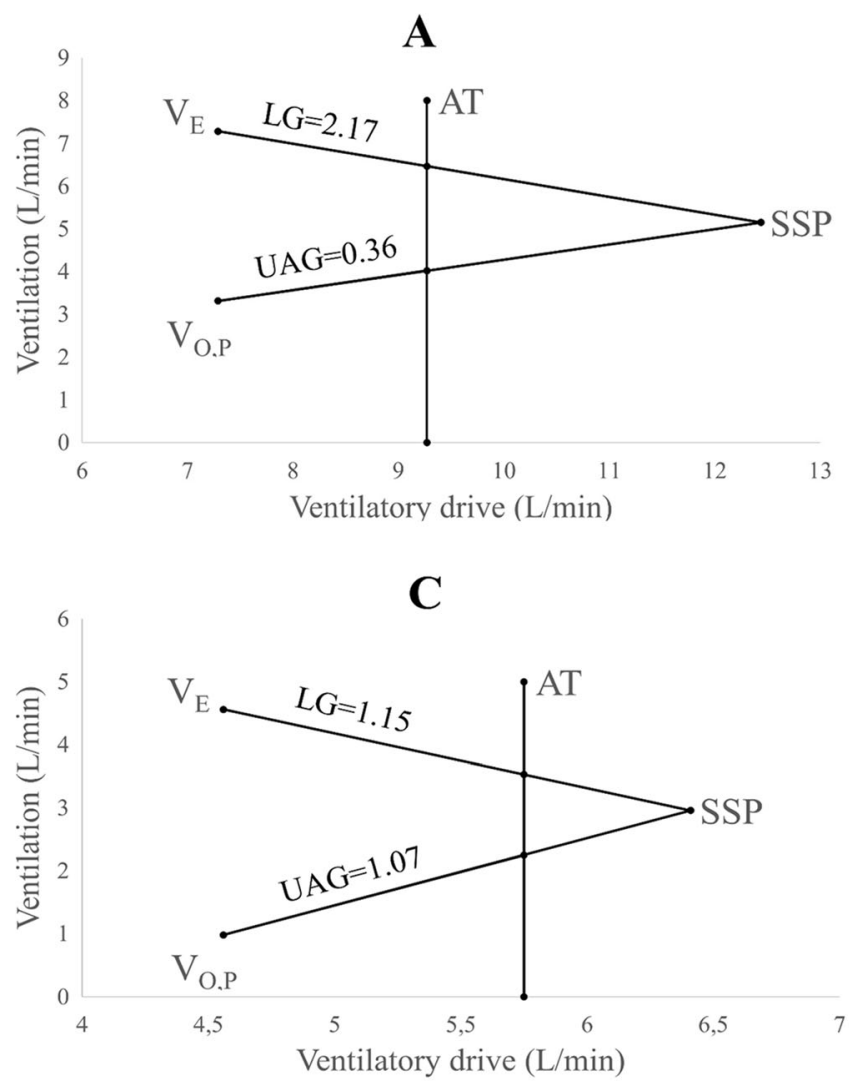

Fig. 1 Patient's phenotyping graph from each treatment arm. a Ventilatory diagram of a representative patient with a low arousal threshold and a high loop gain. We prescribed acetazolamide to decrease the loop gain and trazodone to increase the arousal threshold above the SSP. b Ventilatory diagram of a representative patient with a high loop gain and a very high upper airway collapsibility. We prescribed acetazolamide to decrease the loop gain and compression stockings plus hypocaloric diet to decrease upper airway collapsibility, in order to move the SSP on the left of the arousal threshold line. $\mathbf{c}$ Ventilatory diagram of a ventilation after dial up minus $V_{E}$ (ventilatory overshoot) and the difference between $V_{E}$ and the average ventilation in the last five breaths before dial up (ventilatory disturbance). CPAP dial downs [17] were repeated until at least four reliable measurements of the aforementioned parameters were available for each patient.

Once all these traits were obtained, they were plotted in a graph as average values among the ones obtained from all-night study and following the technique described by Wellman and colleagues [2], where the AT straight line marks the limit between ventilatory stability and instability areas (Fig. 1). Healthy subjects have their steady state point (SSP) of ventilation to the left of the AT line, while OSA patients cannot reach the SSP of ventilation during sleep as it is beyond the arousal threshold line. The distance between AT and SSP in the ventilatory instability area was seen to loosely predict OSA severity [18].
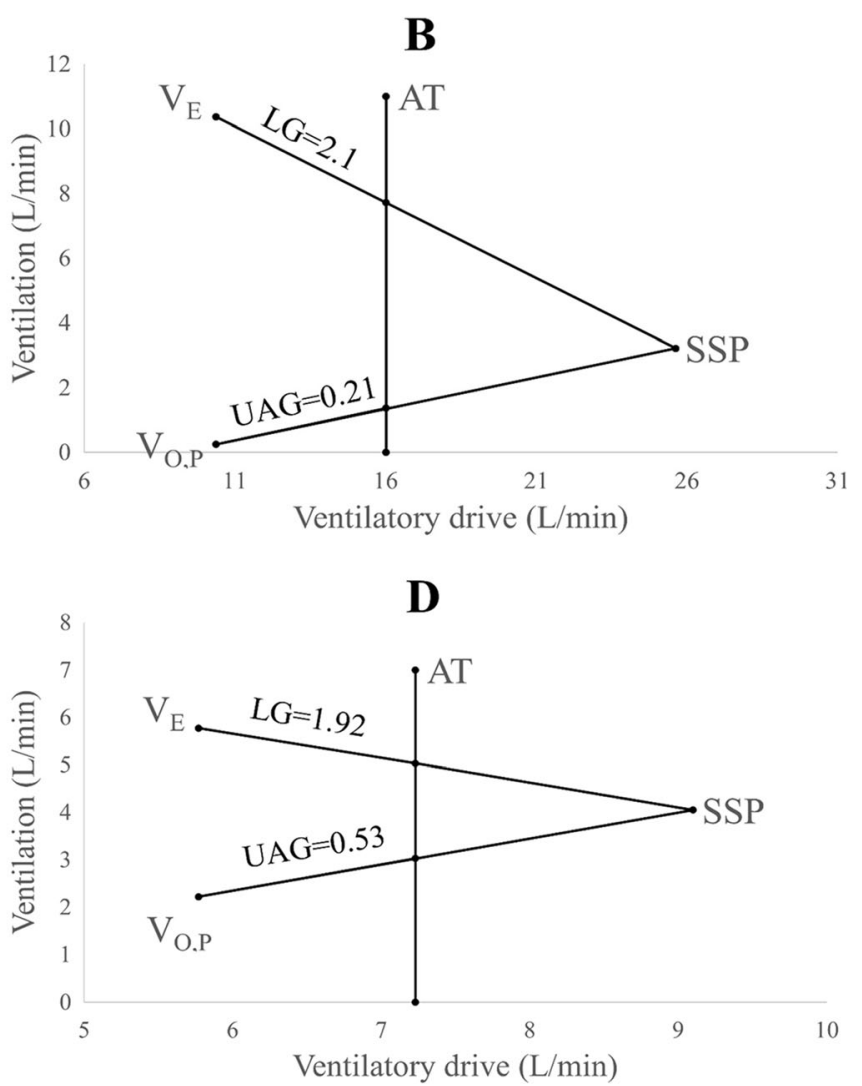

representative patient with a low arousal threshold and a high collapsibility. We prescribed compression stockings to decrease collapsibility and trazodone to increase the arousal threshold above the SSP. d Ventilatory diagram of a representative patient with a low arousal threshold. We prescribed trazodone to increase the arousal threshold above the SSP. $A T$ arousal threshold, $U A G$ upper airway gain, $L G$ loop gain, $V_{O, P}$ mean value of ventilation after having dropped down CPAP from therapeutic level to $0 \mathrm{cmH}_{2} \mathrm{O}, S S P$ steady state point of ventilation, PRE pretreatment data, POST posttreatment data 


\section{Statistics}

Data were analyzed by paired Student's $t$ test, and statistical significance was accepted if $p<0.05$. Data are expressed as mean ( \pm standard deviation). To assess if phenotyping or polysomnographic parameters predicted AHI variability, a stepwise backward multiple regression analysis was performed with the variation between pre- and post-therapy AHI ( $\triangle \mathrm{AHI}$ ) as the dependent variable and $V_{0 \text {,PAssive, }} V_{0, \text { ACTIVE, }}$ LG, and AT as independent variables. Statistical analyses were performed using GraphPad Prism 6.0 (GraphPad Software, La Jolla, California, USA) and SPSS 19.00 (IBM, Armonk, NY, USA).

\section{Results}

Eight subjects, whose anthropometric baseline characteristics are shown in Table 1, were progressively recruited in the study. Patients were middle-aged, overweight, and symptomatic on average. A mean value of 5.5 CPAP dial downs was performed. After phenotyping, four treatment arms were designed. Changes in AHI and ESS score from baseline to post-therapy condition are represented in Fig. 2. Patients had significant reductions in AHI $(p=0.01)$, mostly driven by a drop in apneas $(p=0.02)$, and sleepiness $(p=0.01)$ from pretreatment to post-treatment condition.

Patients experienced only sporadic and minor side effects: rhinorrhea and mild paresthesia likely related to acetazolamide were reported by one patient, while another patient complained a slight increase of daily somnolence that we attributed to trazodone. These mild adverse reactions disappeared at treatment suspension. Even if the side effects were minor, these were present in $25 \%$ of our patients and this fact might affect pharmacological treatment adherence in the long term.

In the first group, patients assumed only trazodone to increase their AT (patient 3 could not take a combined therapy with acetazolamide because of moderate stage kidney failure). After 2 weeks of therapy, AHI, ODI, and somnolence were

Table 1 Anthropometric data and sleepiness questionnaire score for all patients of the study

\begin{tabular}{ll}
\hline & Mean \pm SD \\
\hline Age, years & $66 \pm 12$ \\
BMI, kg/m & $26.9 \pm 4.5$ \\
Neck, cm & $40.5 \pm 5$ \\
Waist, cm & $98 \pm 17$ \\
ESS & $9.5 \pm 4$ \\
\hline
\end{tabular}

Data are expressed as mean (standard deviation)

$B M I$ body mass index, ESS Epworth sleepiness scale, $S D$ standard deviation improved for two patients, while they remained unchanged in one patient.

In the second group, patients undertook a combined pharmacological and behavioral therapy with trazodone and compression stockings to ameliorate their UA collapsibility and AT. There were slight improvements in AHI, ODI, and ESS in both patients, after the therapy period.

In the third group, patients underwent a combined therapy with acetazolamide, compression stockings, and hypocaloric diet, to improve UA collapsibility. The results were good for both of them after the therapy. Particularly, in this subgroup of patients, mean BMI changed from 29.15 to 28.15 , pre-therapy and after therapy, respectively. No significant changes were observed in the whole studied group.

In the last group, there was only one patient, who assumed both acetazolamide and trazodone, with very good results after the therapy.

The mean of individual coefficients of variation for each parameter used to draw the model showed that data were reproducible (mean coefficient of variation for $V_{E}$ was $15 \%$, $34 \%$ for $V_{0, \mathrm{P}}, 39 \%$ for $V_{0, \mathrm{~A}}, 19 \%$ for $V_{\mathrm{AROUSAL}}, 12 \%$ for ventilatory overshoot).

In Table 2, a comparison between polygraphy data before and after therapy is shown. ODI was significantly reduced after therapy as compared to baseline $(p=0.03)$, and, interestingly, it is evident how the reduction in total AHI was mostly driven by a drop in apneic events $(p=0.02)$.

A good relationship between basal AHI and "physiological gap" between $V_{\text {AROUSAL }}$ minus $V_{0, \text { ACTIVE }}(r=0.84, p=0.004)$ was found, in accordance to previous literature [19]. In univariate analysis, the reduction in AHI on therapy was related to baseline AT $(r=0.79, p=0.02)$ and baseline AT-SSP $(r=0.81, p=0.01)$, whereas in multivariate analysis, $\Delta \mathrm{AHI}$ was related only to AT-SSP $(r=0.80, p=0.01)$.

\section{Discussion}

This study demonstrates how a phenotype-based treatment can be effective in reducing AHI and ODI during sleep as well as in leading to an improvement in symptoms in selected OSA patients. To our knowledge, this is the first time that a multidrugs and behavioral approach was proposed, whereas, in a single way, all the drugs we used in this study had already been investigated before.

Indeed, a codified behavioral or pharmacological therapy for OSA does not exist yet, albeit many studies have been performed in these years to find an alternative treatment to CPAP. Schwartz and coworkers first correlated UA collapsibility and body weight [20], attesting how a fall of $6.2 \mathrm{cmH}_{2} \mathrm{O}$ in $P_{\text {CRIT }}$ was matched by a loss of 10 BMI points. It follows that a hypocaloric diet can effectively ameliorate $P_{\text {CRIT }}$ and reduce UA collapsibility. 


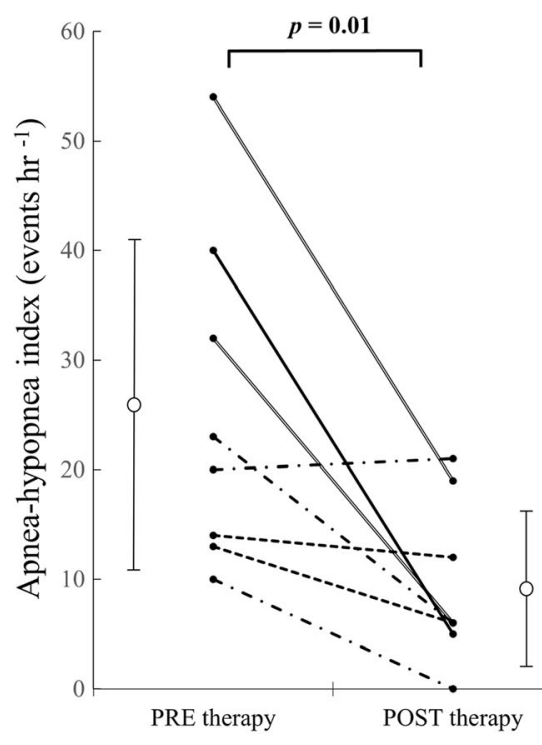

Fig. 2 Individual effects on tailored therapy on the severity of sleepdisordered breathing. Individual data comparing apnea-hypopnea index (AHI) and Epworth sleepiness scale (ESS) at baseline and post-tailored therapy. Changes following treatment with trazodone and acetazolamide are represented as solid line, those following treatment with acetazolamide, compression stockings, and hypocaloric diet are

In recent past years, it has been discussed how rostral fluid shift from legs to the neck due to recumbent position during night can influence UA collapsibility. The amount of fluid shift correlated strongly with AHI and changes in neck circumference in nonobese patients [21], and in a similar population, it is significantly lowered after wearing compression stockings during daytime [22]. This is why we proposed

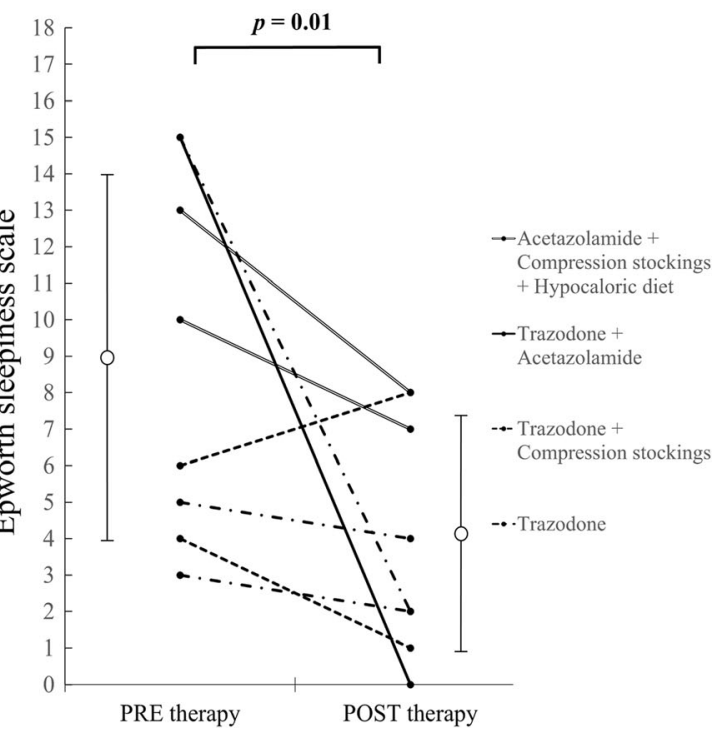

represented as doubled lines, those following treatment with trazodone and compression stockings are represented as dashed lines, and those following treatment with trazodone are represented with point dashed lines. Note as AHI was reduced in all but one subject. Means and standard deviations are represented by a white circle and vertical bars, respectively

hypocaloric diet and compression stockings during daytime in patients whose UA were highly collapsible.

A number of drugs have been proposed to treat sleep apnea. In 2008, Heinzer and colleagues tried to administrate trazodone, a nonmyorelaxant sleep-inducing drug able to raise serotonin levels in the central nervous system, to OSA patients. They found the drug to be related to an increase of $48 \%$ of
Table 2 Comparison between before and after therapy polygraphy data

\begin{tabular}{llll}
\hline & Before therapy & After therapy & $p$ \\
\hline Total AHI, events/h & $26(15)$ & $9(7)$ & 0.01 \\
Supine AHI, events/h & $25(16.5)$ & $13(9)$ & 0.1 \\
Nonsupine AHI, events/h & $12.5(18.5)$ & $4(7)$ & 0.11 \\
ODI, events/h & $22(15)$ & $9(8)$ & 0.03 \\
Supine ODI, events/h & $22(16)$ & $11.5(10)$ & 0.14 \\
Nonsupine ODI, events/h & $11.5(18)$ & $4(6)$ & 0.14 \\
Supine sleep time, \%total sleep time & $58(37)$ & $57(26)$ & 0.92 \\
Nonsupine sleep time, $\%$ total sleep time & $42(36)$ & $43(26)$ & 0.94 \\
Apnea Index, events/h & $15(11)$ & $3(3)$ & 0.02 \\
Hypopnea Index, events/h & $10(8)$ & $6(6.5)$ & 0.2 \\
$\mathrm{MeanSaO}_{2}, \%$ & $93.5(1.5)$ & $94.5(2)$ & 0.2 \\
$\mathrm{MinSaO}_{2}, \%$ & $81(7)$ & $82(6)$ & 0.35 \\
$\mathrm{MeanDeSaO}_{2}, \%$ & $88(5)$ & $91(2)$ & 0.27 \\
$\mathrm{CT} 90, \%$ & $6.7(7)$ & $7(11)$ & 0.95 \\
\hline
\end{tabular}

Data are expressed as mean (standard deviation)

$\mathrm{ODI}$ Oxygen Desaturation Index, $\mathrm{MeanSaO}_{2}$ average nocturnal oxyhemoglobin saturation, $\mathrm{MinSaO}_{2}$ lowest nocturnal oxyhemoglobin saturation, $\mathrm{MeanDeSaO}$ average nocturnal oxyhemoglobin desaturation, CT90\% analysis time with $\mathrm{SaO}_{2}<90 \%$, n.s. not significant 
arousal threshold in response to hypercapnia, and this mechanism allowed OSA patients to accumulate a higher level of $\mathrm{CO}_{2}$ without awaking. It had been previously postulated that OSA patients with a high arousal threshold can stay asleep enough to recruit UA dilatator muscles and to maintain opened UA, reaching a pattern of stable ventilation [10,23].

Wellman et al. first introduced the OSA phenotyping approach and proposed the treatment based on a personalized model [2]. Then, many drug trials were performed to understand how to modify each trait of that model and trazodone was deeply investigated. Eckert and coworkers [24] showed that $100 \mathrm{mg}$ of trazodone increased significantly the arousal threshold in patients with sleep apnea and a low arousal threshold without affecting UA dilator muscle activity. Despite the increased arousal threshold, the AHI in that study was unchanged compared to placebo. On the contrary, in the study of Smales and coworkers [25], AHI of 15 unselected OSA patients was found significantly decreased after the administration of trazodone $100 \mathrm{mg}$ as compared to placebo, showing that this drug could be an effective therapy for some patients with OSA. Loop gain can be reduced by using acetazolamide, a carbonic anhydrase inhibitor. Wellman et al. [26] found that acetazolamide $500 \mathrm{mg}$, administered twice daily for a week, decreased LG by $41 \%$ by lowering plant-gain and attenuating the ventilatory response to spontaneous arousals [27] with a concomitant reduction of AHI by $47 \%$ in OSA patients. In selected OSA patients, UAG can be improved with hypoglossal nerve stimulation [28] or with desipramine [29]. However, the former requires wide economic accessibility and dedicated centers, while the latter requires more studies in OSA patients. Finally, Edwards and colleagues innovatively tested a combination therapy with supplemental oxygen and Eszopiclone $3 \mathrm{mg}$ in 20 nonselected OSA subjects, obtaining reduction by more than $50 \%$ of obstructive events in 9 patients, those with favorable anatomy and a small difference between $V_{\text {AROUSAL }}$ and $V_{0, \text { ACTIVE }}$, and in any case improving AHI in another $50 \%$ of that population [19].

According to the abovementioned findings, we administered trazodone to those who had a low AT, acetazolamide to those who had a high LG, and behavioral therapy to those who had an increased $P_{\text {CRIT }}$. However, differently from previous studies, we offered a composite treatment adopting either drugs or behavioral approach every time the corresponding altered pathogenic trait was found. Furthermore, differently from existing literature, our patients were naïve from CPAP and this encourages us to believe that phenotypes were not affected by previous ventilator usage (i.e., decreasing in both LG and AT) [30], even if, for the same reason, we cannot exclude that alterations either in LG or AT might be a consequence of OSA itself. However, given that most of the subjects responded well to our treatments, the nonanatomic traits had more likely a causative role. By obtaining a $65 \%$ reduction in mean total AHI, mostly via a reduction in obstructive apneas, a 59\% reduction in mean total ODI and a 55\% reduction in mean ESS score from baseline, we demonstrated that this model can effectively improve OSA severity and the associated symptoms.

This study presents some limitations. First of all, the lack of a control arm and the small number of patients allows us only to draw preliminary conclusions about efficacy of composite treatment administered according to such approach, and this field clearly needs and deserves further investigations to conceivably extend the findings to the whole OSA population. Secondly, the impossibility to run a second physiology night after the therapy does not give us certainties regarding whether or not a pathogenic trait had been really modified. It would have been desirable to make a comparison between phenotypic traits before and after the treatment to be sure the reductions in AHI were provoked by phenotypic-based treatment amelioration. Furthermore, to detect variations, if any, in apnea onset during NREM and REM sleep, accounting on the difference in prevalence between the two categories of OSA, performing a complete polysomnography pre and after therapy would have been of even greater utility [31]. Moreover, it cannot be excluded that our results might be the effect of night-to-night variability [32, 33]. Regarding fluid shift, we did not measure calf circumferences and we do not have data on waist and neck circumferences after treatments to better clarify the link between improvement in AHI and reduction in fluid shift. Besides this, it would have been worth to analyze the effect of tailored therapy through a longer period of time, better estimating the benefitrisk ratio for patients, most of all for who was diagnosed with a mild-to-moderate OSA, and the direct impact of hypocaloric diet on UA collapsibility. Finally, we drafted phenotypes basing the analyses only on NREM sleep data, and we cannot ignore that pathogenetic traits could be very different because of several reasons, i.e., diminished LG and reduced UAG, during REM sleep $[34,35]$. In turn, this might explain why certain subjects had better responses as compared to others.

\section{Conclusions}

A personalized therapy in phenotyped OSA patients may induce a remarkable improvement in AHI, ODI, and sleepiness. OSA phenotyping would be desirable to become a routinely practice in order to offer OSA patients an alternative treatment to CPAP and to further investigate drug effects which could interact with the pathophysiology of this disease.

Author contributions Dr. Messineo contributed to study design, data collection, data analysis and interpretation, and drafting and review of the manuscript for important intellectual content. Dr. Magri contributed to the study design, data collection and interpretation, and drafting and review of the manuscript for important intellectual content. Dr. Corda contributed to data collection and review of the manuscript. Dr. Pini contributed to data collection and review of the manuscript. Dr. Taranto-Montemurro 
contributed to data analysis and interpretation, and drafting and review of the manuscript for important intellectual content. Dr. Tantucci contributed to the final approval of the version submitted for publication, study design, data analysis and interpretation, and review of the manuscript for important intellectual content

\section{Compliance with ethical standards}

Fundings No funding was received for this research.

Conflict of interests Luigi Taranto-Montemurro reports personal fees from Novion Pharmaceuticals, outside the submitted work.

The other authors certify that they have no affiliation with or involvement in any organization or entity with any financial interest.

Ethical approval All procedures performed in studies involving human participants were in accordance with the ethical standards of institutional and/or national research committee and with the 1964 Helsinki declaration and its later amendments or comparable ethical standards.

Informed consent Informed consent was obtained from all individual participants included in the study.

\section{References}

1. Franklin K, Lindberg E (2015) Obstructive sleep apnea is a common disorder in the population-a review on the epidemiology of sleep apnea. J Thorac Dis 7(8):1311-1322

2. Wellman A, Eckert D, Jordan A, Edwards B, Passaglia C, Jackson A, Gautam S, Owens R, Malhotra A, White D (2011) A method for measuring and modeling the physiological raits causing obstructive sleep apnea. J Appl Physiol 110(6):1627-1637

3. Gleadhill I, Schwartz A, Wise R, Permutt S, Smith P (1991) Upper airway collabsibility in snorers and in patients with obstructive hypopnea and apnea. Am Rev Respir Dis 43:1300-1303

4. Isono S, Remmers J, Tanaka A, Sho Y (1997) Anatomy of pharynx in patients with obstructive sleep apnea and in normal subjects. J Appl Physiol 82:1319-1326

5. Campana L, Eckert D, Patel S, Malhotra A (2010) Pathophysiology \& genetics of obstructive sleep apnoea. Indian J Med Res 131:176187

6. Pharm L, Schwartz A (2015) The pathogenesis of obstructive sleep apnea. J Thorac Dis 7(8):1358-1372

7. Taranto Montemurro L, Bettinzoli M, Corda L, Redolfi S, Novali M, Braghini A, Tantucci C (2012) Influence of upper airway size on volume exhaled under negative pressure during evaluation of upper airway collapsibility. Sleep Breath 16(2):399-404. doi:10.1007/ s11325-011-0511-3

8. Patil S, Schneider H, Marx J, Gladmon E, Schwartz A, Smith P (2007) Neuromechanical control of upper airway patency during sleep. J Appl Physiol 102:547-556

9. Eckert D, Lo Y, Saboisky J, Jordan A, White D, Malhotra A (2011) Sensorimotor function of the upper-airway muscles and respiratory sensory processing in untreated obstructive sleep apnea. J Appl Physiol 11:1644-1653

10. Younes M (2004) Role of arousals in the pathogenesis of obstructive sleep apnea. Am J Respir Crit Care Med 169:623-633

11. Younes M, Ostrowski M, Thompson W, Leslie C, Shewchuk W (2001) Chemical control stability in patients with obstructive sleep apnea. Am J Respir Crit Care Med 163:1181-1190

12. Wellman A, Jordan A, Malhotra A, Fogel R, Katz E, Schory K, Edwards J, White D (2001) Ventilatory control and airway anatomy in obstructive sleep apnea. Am J Respir Crit Care Med 170:12251232

13. Weaver T, Grunstein T (2008) Adherence to continuous positive airway pressure: the challenge to effective treatment. Proc Am Thorac Soc 15(5):173-178

14. Wolkove N, Baltzan M, Kamel H, Dabrusin R, Palayew M (2008) Long-term compliance with continuous positive airway pressure in patients with obstructive sleep apnea. Can Respir J 15(7):365-369

15. Eckert D, White D, Jordan A, Malhotra A, Wellman A (2013) Defining phenotypic causes of obstructive sleep apnea. Identification of novel therapeutic targets. Am J Respir Crit Care Med 188(8):996-1004

16. Berry R, Budhiraja R, Gottlieb D, Gozal D, Iber C, Kapur V et al (2012) Rules for scoring respiratory events in sleep: update of the 2007 AASM manual for the scoring of sleep and associated events. Deliberations of the sleep apnea definitions task force of the American Academy of sleep medicine. J Clin Sleep Med 8(5): 597-619

17. Wellman A, Edwards E, Sands S, Owens R, Butler S, Passaglia C, Jackson A, Malhotra A, White D (2013) A simplified method for determining phenotypic traits in patients with obstructive sleep apnea. J App Physiol 114:911-922

18. Owens R, Edwards B, Eckert D, Jordan A, Sands S, Malhotra A, White D, Loring S, Butler J, Wellman A (2015) An integrative model of physiological traits can be used to predict obstructive sleep apnea and response to non positive airway pressure therapy. Sleep 38(6):961-970

19. Edwards B, Sands S, Owens R, Eckert D, Landry S, White D, Malhotra A, Wellman A (2016) The combination of supplemental oxygen and a hypnotic markedly improves obstructive sleep apnea in patients with a mild to moderate upper airway collapsibility. Sleep 39(11):1973-1983

20. Schwartz A, Gold A, Schubert N, Stryzak A, Wise R, Permutt S, Smith P (1991) Effect of weight loss on upper airway collapsibility in obstructive sleep apnea. Am Rev Respir Dis 144(3):494-498

21. Redolfi S, Yumino D, Ruttanaumpawan P, Yau B, Su M, Lam J, Bradley T (2009) Relationship between overnight rostral fluid shift and obstructive sleep apnea in nonobese men. Am J Respir Crit Care Med 173(3):241-246

22. Redolfi S, Arnulf I, Pottier M, Bradley T, Similowski T (2011) Effects of compression of the legs on overnight rostral fluid shift and obstructive sleep apnea. Respir Physiol Neurobiol 175(3):390 393

23. Jordan A, White D, Lo Y, Wellman A, Eckert D, Yim-Yeh S, Eikermann M, Smith S, Stevenson K, Malhotra A (2009) Airway dilator muscle activity and lung volume during stable breathing in obstructive sleep apnea. Sleep 32:361-368

24. Eckert D, Malhotra A, Wellman A, White D (2014) Trazodone increases the respiratory arousal threshold in patients with obstructive sleep apnea and a low arousal threshold. Sleep 37(4):811-819

25. Smales E, Edwards B, Deyoung P, McSharry D, Wellman A, Velasquez A, Owens R, Orr J, Malhotra A (2015) Trazodone effects on obstructive sleep apnea and NON-REM arousal threshold. Ann Am Thorac Soc 12(5):758-764

26. Edwards B, Sands S, Eckert D, White D, Butler J, Owens R, Malhotra A, Wellman A (2012) Acetazolamide improves loop gain but not the other physiological traits causing obstructive sleep apnea. J Physiol 590(5):1199-1211

27. Edwards B, Connolly J, Campana L, Sands S, Trinder J, White D, Wellman A, Malhotra A (2013) Acetazolamide attenuates the ventilatory response to arousal in patients with obstructive sleep apnea. Sleep 36(2):281-285

28. Kezirian E, Goding GJ, Malhotra A, O'Donoghue F, Zammit G, Wheatley J, Catcheside P, Smith P, Schwartz A, Walsh J, Maddison K, Claman C, Huntley T, Park S, Campbell M, Palme C, Iber C, Eastwood P, Hillman D, Barnes M (2014) Hypoglossal 
nerve stimulation improves obstructive sleep apnea; 12 -month outcomes. J Sleep Res 23(1):77-83

29. Taranto-Montemurro L, Edwards B, Sands S, Marques M, Eckert D, White D, Wellman A (2016) Desipramine increases genioglossus activity and reduces upper airway collapsibility during non-REM sleep in healthy subjects. Am J Respir Crit Care Med. doi:10.1164/rccm.201511-2172OC

30. Loewen A, Ostrowski M, Laprairie J, Atkar R, Gnitecki J, Hanly P, Younes M (2009) Determinants of ventilatory instability in obstructive sleep apnea: inherent or acquired? Sleep 32(10):1355-1365

31. Koo B, Dostal J, Ioachimescu O, Budur K (2008) The effect of gender and age on REM-related sleep-disordered breathing. Sleep Breath 12(3):259-264

32. Levendowski D, Zack N, Rao S, Wong K, Gendreau M, Kranzler J, Zavora T, Westbrook P (2009) Assessment of the test-retest reliability of laboratory polysomnography. Sleep Breath 13(2):163-167

33. Newell J, Mairesse O, Verbanck P, Neu D (2012) Is a one-night stay in the lab really enough to conclude? First-night effect and night-to- night variability in polysomnographic recordings among different clinical population samples. Psychiatry Res 200:795-801

34. Sands S, Owens R (2015) Congestive heart failure and central sleep apnea. Crit Care Clin 31(3):473-495

35. McSharry D, Saboisky J, Deyoung P, Jordan A, Trinder J, Smales E, Hess L, Chamberlin N, Malhotra A (2014) Physiological mechanisms of upper airway hypotonia during REM sleep. Sleep Breath 37(3):561-569

\section{Comment}

This is the first time that the Wellman/Brigham and Women's/Harvard phenotyping method has been put into clinical practice by another lab. It shows the promise and potential limitations of this approach.

Robert Owens

Boston, USA 